\title{
Generalized Jordan Semitriple Maps on Hilbert Space Effect Algebras
}

\author{
Qing Yuan and Kan He \\ College of Mathematics, Institute of Mathematics, Taiyuan University of Technology, Taiyuan, Shanxi 030024, China \\ Correspondence should be addressed to Kan He; hk19830310@163.com
}

Received 31 December 2013; Accepted 20 March 2014; Published 13 April 2014

Academic Editor: Andrei D. Mironov

Copyright (C) 2014 Q. Yuan and K. He. This is an open access article distributed under the Creative Commons Attribution License, which permits unrestricted use, distribution, and reproduction in any medium, provided the original work is properly cited.

Let $\mathscr{E}(H)$ be the Hilbert space effect algebra on a Hilbert space $H$ with $\operatorname{dim} H \geq 3, \alpha, \beta$ two positive numbers with $2 \alpha+\beta \neq 1$ and $\Phi: \mathscr{E}(H) \rightarrow \mathscr{E}(H)$ a bijective map. We show that if $\Phi\left(A^{\alpha} B^{\beta} A^{\alpha}\right)=\Phi(A)^{\alpha} \Phi(B)^{\beta} \Phi(A)^{\alpha}$ holds for all $A, B \in \mathscr{E}(H)$, then there exists a unitary or an antiunitary operator $U$ on $H$ such that $\Phi(A)=U A U^{*}$ for every $A \in \mathscr{E}(H)$.

\section{Introduction and Notations}

Let $H$ be a Hilbert space. Denote by $\mathscr{B}(H)$ the algebra of all bounded linear operators acting on $H$. The operator interval in $\mathscr{B}(H): \mathscr{E}(H)=\{T \in \mathscr{B}(H) \mid 0 \leq T \leq$ $I\}$, where $I$ is the identity, is called Hilbert space effect algebra on $H$, and the elements in $\mathscr{E}(H)$ are called effects. The concept of effect algebras plays a fundamental role in mathematical description of quantum measurement, and the range of general quantum observables (POV-measurement) consists of effects [1].

The Hilbert space effect algebra $\mathscr{E}(H)$ can be equipped with some algebraic operations. Denote the space of all projections on $H$ by $\mathscr{P}(H)$. Clearly $\mathscr{P}(H) \subseteq \mathscr{E}(H)$. Let $A, B \in$ $\mathscr{E}(H) . \mathscr{E}(H)$ is a partial ordered set with the order " $\leq ": A \leq B$ if $B-A \geq 0$. Obviously, the sum $A+B$ may not be in $\mathscr{E}(H)$. In the case that $A+B \in \mathscr{E}(H)$, we can define an operation of conditional addition in $\mathscr{E}(H): A \oplus B=A+B$. Particularly, since $P+Q \in \mathscr{P}(H) \Leftrightarrow P Q=0$, this gives an orthogonal addition operation in $\mathscr{P}(H)$. Generally speaking, the product $A B$ may not be in $\mathscr{E}(H)$ either. However, the Jordan semitriple product $A \circ B=A B A$ is a well-defined operation in $\mathscr{E}(H)$ since $A B A \in \mathscr{E}(H)$ for all $A, B \in \mathscr{E}(H)$. More generally, for any fixed positive real numbers $\alpha, \beta$, the generalized Jordan triple product ${ }_{\alpha, \beta}$ induced by $(\alpha, \beta)$ is well defined, too, in $\mathscr{E}(H)$ and here $A_{\alpha, \beta} B=A^{\alpha} B^{\beta} A^{\alpha}$.
Recently many mathematicians pay their attention to the problem of characterizing certain maps on Hilbert space effect algebras or other quantum structures [2-8]. Let $H$ be a Hilbert space with $\operatorname{dim} H \geq 3$ and $\Phi: \mathscr{E}(H) \rightarrow \mathscr{E}(H)$ a bijective map. It seems that [5] is the first paper discussing the problem of characterizing the Jordan semitriple maps on Hilbert effect algebras. In [5] Molnár showed that if $\Phi(A B A)=\Phi(A) \Phi(B) \Phi(A)$ for all $A, B \in \mathscr{E}(H)$, then there exists a unitary or an antiunitary operator $U$ on $H$ such that $\Phi(A)=U A U^{*}$ for every $A \in \mathscr{E}(H)$; that is, $\Phi$ is implemented by a unitary or an antiunitary operator on $H$. Molnár and Šemrl proved in [7] that if $\Phi$ satisfies

$$
\Psi\left(A \circ_{1 / 2,1} B\right)=\Psi(A) \circ_{1 / 2,1} \Psi(B) \quad \forall A, B \in \mathscr{E}(H),
$$

where $\Psi$ denotes $\Phi$ and $\Phi^{-1}$, then there is a positive number $p$ and a unitary or an antiunitary operator $U$ such that $\Phi(A)=$ $U A^{p} U^{*}$ for every $A \in \mathscr{E}(H)$ (we will show that $p=1$ in fact). The generalized Jordan semitriple product $A \circ_{1 / 2,1} B=$ $A^{1 / 2} B A^{1 / 2}$ is called sequence product. Kim showed in [4] that if $H$ is separable and $\Phi$ satisfies

$$
\begin{gathered}
A B=B A \Longrightarrow \Phi(A B)=\Phi(A) \Phi(B)=\Phi(B) \Phi(A), \\
A B=B A, \\
A+B \in \mathscr{E}(H) \Longrightarrow \Phi(A+B)=\Phi(A)+\Phi(B)
\end{gathered}
$$


for $A, B \in \mathscr{E}(H)$, then $\Phi$ is also implemented by a unitary or an antiunitary operator on $H$. Our purpose in this paper is to generalize the results of $[5,7]$ to generalized Jordan semitriple maps, that is, the maps $\Phi$ satisfying

$$
\Phi\left(A \circ_{\alpha, \beta} B\right)=\Phi(A){ }_{\alpha, \beta} \Phi(B)
$$

Let $H$ be a Hilbert space with $\operatorname{dim} H \geq 3, \alpha, \beta$ two positive real numbers with $2 \alpha+\beta \neq 1$, and $\Phi: \mathscr{E}(H) \rightarrow \mathscr{E}(H)$ a bijective map satisfying

$$
\Phi\left(A^{\alpha} B^{\beta} A^{\alpha}\right)=\Phi(A)^{\alpha} \Phi(B)^{\beta} \Phi(A)^{\alpha}
$$

for all $A, B \in \mathscr{E}(H)$. We first show that $\Phi$ is orthoadditive on $\mathscr{P}(H)$, that is, for any orthogonal projections $P, Q, \Phi(P+Q)=$ $\Phi(P)+\Phi(Q)$. As an application, we prove that all bijective generalized Jordan semitriple maps on Hilbert space effect algebras, that is, the maps satisfying (4), are implemented by a unitary or an antiunitary operator on $H$.

\section{Orthoadditivity of Generalized Jordan Semitriple Maps}

Additivity of multiplicative maps is studied by many authors. In this section, we show that every generalized Jordan semitriple map on the effect algebra $\mathscr{E}(H)$ is orthoadditive on $\mathscr{P}(H)$. To do this we need a description which gives a description of the zero product preservers on $\mathscr{P}(H)$. Recall that a map $\Psi$ preserves zero products (in both directions) if $\Psi(A) \Psi(B)=0$, whenever (if and only if) $A B=0$.

Lemma 1. Let $H$ be a real or complex Hilbert space with $\operatorname{dim} H \geq 3$. Assume that $\Phi: \mathscr{P}(H) \rightarrow \mathscr{P}(H)$ is a bijective map preserving zero products of operators in both directions.

(1) If $H$ is real, then there is a unitary operator $U$ on $H$ such that $\Phi(P)=U P U^{*}$ for all rank one $P \in \mathscr{P}(H)$.

(2) If $H$ is complex, then there is a unitary operator or an antiunitary operator $U$ on $H$ such that $\Phi(P)=U P U^{*}$ for all rank one $P \in \mathscr{P}(H)$.

Proof. Denote by $\mathscr{P}_{1}(H)$ the set of all projections of rank one. For any $P \in \mathscr{P}(H)$, write $\{P\}^{\perp}=\{T \in \mathscr{P}(H): T \neq 0, T P=0\}$.

Claim 1. For any $P \in \mathscr{P}(H),\{P\}^{\perp}=\emptyset$ if and only if $P=I$; $\{P\}^{\perp}$ is singleton; that is, it contains exactly one element, if and only if $I-P$ is of rank one.

If $P=I \Rightarrow\{P\}^{\perp}=\emptyset$ is obvious, assume that $P \neq I$ and then $I-P \neq 0$ and $I-P \in\{P\}^{\perp}$.

If $\{P\}^{\perp}$ contains only one element, then $P \neq I$ and $\{P\}^{\perp}=$ $\{I-P\}$. If $I-P$ is not of rank one, for any unit vector $x \in \operatorname{ran}(I-$ $P$ ), one has $x \otimes x \in\{P\}^{\perp}$ but $x \otimes x \neq I-P$, a contradiction. So, $\operatorname{rank}(I-P)=1$. The converse is obvious.

Claim 2. $\Phi$ preserves rank one projections in both directions.

For any rank one projection $x \otimes x$, let $P=I-x \otimes x$; then $\{x \otimes$ $x\}=\{P\}^{\perp}$. By the property of $\Phi$ that it preserves zero products in both directions, one has $\Phi(\{x \otimes x\})=\Phi\left(\{P\}^{\perp}\right)=\{\Phi(P)\}^{\perp}$.
Thus $\{\Phi(P)\}^{\perp}$ is singleton, which forces that $\Phi(x \otimes x)$ is of rank one by Claim 1 , and vice versa.

Claim 3. There exists a unitary or antiunitary operator $U$ on $H$ such that

$$
\Phi(P)=U P U^{*}
$$

holds for all $P \in \mathscr{P}_{1}(H)$. $U$ is unitary in real case.

By Claim 3, for every unit vector $x \in H$, there is a unit vector $y_{x} \in H$ such that $\Phi(x \otimes x)=y_{x} \otimes y_{x}$.

Let $\phi: P H \rightarrow P H$ be the map on the projective space PH defined by $\phi([x])=\left[y_{x}\right]$, where $[x]$ stands for the 1 dimensional linear subspace spanned by $x$. Then it is clear that $\phi$ is bijective since $\Phi$ preserves rank one projection in both directions. For any linearly independent unit vectors $x, u$ and any scalars $\gamma, \delta$, let $v=\|\gamma x+\delta u\|^{-1}(\gamma x+\delta u)$. For any unit $y \in H$, there is a unit vector $w$ such that $\Phi(w \otimes w)=$ $y \otimes y$. If $\left\langle y, y_{x}\right\rangle=\left\langle y, y_{u}\right\rangle=0$, then $(y \otimes y)\left(y_{x} \otimes y_{x}\right)=$ $(y \otimes y)\left(y_{x} \otimes y_{x}\right)=0$. This implies that $\langle w, x\rangle=\langle w, u\rangle=0$ and consequently $\langle w, v\rangle=0$. Hence $\left\langle y, y_{v}\right\rangle=0$. It follows from the arbitrariness of $y$ that $y_{v} \in\left[y_{x}\right]+\left[y_{u}\right]$; that is, $[v] \in[x]+[u] \Rightarrow \phi(v) \in \phi(x)+\phi(u)$. Now we can use the fundamental theorem of projective geometry to get a semilinear bijection $A: H \rightarrow H$ such that $\phi([x])=[A x]$. Therefore, for any unit $x \in H, \Phi(x \otimes x)=\|A x\|^{-2} A x \otimes A x$. It is obvious that $A$ preserves orthogonality in both directions; that is, $\langle x, u\rangle=0 \Leftrightarrow\langle A x, A u\rangle=0$. Hence $A=c U$ for some unitary or antiunitary operator $U$ on $H$. In the case that $H$ is real, $U$ is unitary. Clearly, $|c|=1$, so $\Phi(x \otimes x)=U(x \otimes x) U^{*}$ for all rank one projection $x \otimes x$.

Theorem 2. Let $H$ be a Hilbert space with $\operatorname{dim} H \geq 3, \alpha, \beta$ positive numbers with $2 \alpha+\beta \neq 1$, and $\Phi: \mathscr{E}(H) \rightarrow \mathscr{E}(H) a$ bijective map. If $\Phi$ satisfies

$$
\Phi\left(A^{\alpha} B^{\beta} A^{\alpha}\right)=\Phi(A)^{\alpha} \Phi(B)^{\beta} \Phi(A)^{\alpha},
$$

for all $A, B \in \mathscr{E}(H)$, then for any projections $P, Q, P \perp Q$ implies that

$$
\Phi(P+Q)=\Phi(P)+\Phi(Q)
$$

Proof. For $P \in \mathscr{P}(H)$, we have

$$
\Phi(P)^{\gamma}=\Phi(P)
$$

where $\gamma>0$. Note $\Phi(P) \geq 0$. For $\gamma \neq 1$, the spectral solution theorem implies that $\Phi(P)^{2}=\Phi(P)$. By considering $\Phi^{-1}$, we see that $\Phi$ preserves projections in both directions.

For any $A \in \mathscr{E}(H)$,

$$
\begin{aligned}
\Phi(I) \Phi(A) & =\Phi(I) \Phi(I A I)=\Phi(I) \Phi\left(I^{\alpha}\left(A^{1 / \beta}\right)^{\beta} I^{\alpha}\right) \\
& =\Phi(I) \Phi(I)^{\alpha} \Phi\left(A^{1 / \beta}\right)^{\beta} \Phi(I)^{\alpha} \\
& =\Phi(I) \Phi\left(A^{1 / \beta}\right)^{\beta} \Phi(I) .
\end{aligned}
$$


Similarly, we have

$$
\Phi(I) \Phi(A)=\Phi(I) \Phi\left(A^{1 / \beta}\right)^{\beta} \Phi(I) .
$$

So

$$
\Phi(A) \Phi(I)=\Phi(I) \Phi(A)
$$

holds for all $A \in \mathscr{E}(H)$. By the surjectivity of $\Phi$ and $\Phi(I)=$ $\Phi(I)^{2}$, we must have $\Phi(I)=I$. Since $\Phi$ is bijective, there is $A_{0} \in \mathscr{E}(H)$ such that $\Phi\left(A_{0}\right)=0$. Thus

$$
\Phi(0)=\Phi\left(A_{0}^{\alpha} 0 A_{0}^{\alpha}\right)=\Phi\left(A_{0}\right)^{\alpha} \Phi(0)^{\beta} \Phi\left(A_{0}\right)^{\alpha}=0 ;
$$

that is, $\Phi(0)=0$.

Now we show that $\Phi$ preserves zero product in both directions on $\mathscr{P}(H)$, that it preserves orthogonality in both directions. For any $P, Q$ in $\mathscr{P}(H)$,

$$
\begin{aligned}
P Q & =0 \Longleftrightarrow P^{\beta / 2} Q^{\alpha}=0 \Longleftrightarrow\left(P^{\beta / 2} Q^{\alpha}\right)^{*} P^{\beta / 2} Q^{\alpha} \\
& =0 \Longleftrightarrow Q^{\alpha} P^{\beta} Q^{\alpha}=0 .
\end{aligned}
$$

Then

$$
\begin{aligned}
P Q & =0 \Longleftrightarrow Q^{\alpha} P^{\beta} Q^{\alpha}=0 \Longleftrightarrow \Phi\left(Q^{\alpha} P^{\beta} Q^{\alpha}\right)=0 \\
& \Longleftrightarrow \Phi(Q)^{\alpha} \Phi(P)^{\beta} \Phi(Q)^{\alpha}=0 \Longleftrightarrow \Phi(P) \Phi(Q)=0 .
\end{aligned}
$$

Therefore, by Lemma 1, there exists a unitary or an antiunitary operator $U$ on $H$ such that $\Phi(P)=U P U^{*}$ holds for all rank one projections $P$. Without loss of generality we may assume that $\Phi(P)=P$ for every rank one projection $P$.

For any $P, Q \in \mathscr{P}(H)$, if $P \perp Q$, let $R=\Phi(P+Q) \in$ $\mathscr{P}(H), S=\Phi(P) \in \mathscr{P}(H)$, and $T=\Phi(Q) \in \mathscr{P}(H)$. We will show that $R=S+T$. To do this, it is enough to show that $\operatorname{ran}(R)=\operatorname{ran}(S+T)$. Note that for any $x$ in $H$ with norm 1 ,

$$
\begin{aligned}
x \otimes x R x \otimes x & =(x \otimes x)^{\alpha} R^{\beta}(x \otimes x)^{\alpha} \\
& =\Phi\left((x \otimes x)^{\alpha}(P+Q)^{\beta}(x \otimes x)^{\alpha}\right) \\
& =\Phi(x \otimes x(P+Q) x \otimes x), \\
& x \otimes x \perp S \Longleftrightarrow x \otimes x \perp P, \\
& x \otimes x \perp T \Longleftrightarrow x \otimes x \perp Q .
\end{aligned}
$$

Thus we have

$$
\begin{aligned}
x \otimes x R x \otimes x & =0 \Longleftrightarrow x \otimes x(P+Q) x \otimes x \\
& =0 \Longleftrightarrow x \otimes x(S+T) x \otimes x=0 .
\end{aligned}
$$

This forces $\operatorname{ran}(R)=\operatorname{ran}(S+T)$. So $\Phi(P+Q)=\Phi(P)+\Phi(Q)$ whenever $P \perp Q$.

\section{Characterization of Generalized Jordan Semitriple Maps}

The following is our main result.
Theorem 3. Let $H$ be a Hilbert space with $\operatorname{dim} H \geq 3$. Assume that $\alpha, \beta$ are positive numbers with $2 \alpha+\beta \neq 1$ and $\Phi: \mathscr{E}(H) \rightarrow \mathscr{E}(H)$ a bijective map. If

$$
\Phi\left(A^{\alpha} B^{\beta} A^{\alpha}\right)=\Phi(A)^{\alpha} \Phi(B)^{\beta} \Phi(A)^{\alpha},
$$

for all $A, B \in \mathscr{E}(H)$, then there exists a unitary or an antiunitary operator $U$ on $H$ such that $\Phi(A)=U A U^{*}$ for all $A \in \mathscr{E}(H)$.

The following corollary gives a better form of sequence product multiplicative maps, comparing with [7].

Corollary 4. Let $H$ be a Hilbert space with $\operatorname{dim} H \geq 3$ and $\Phi: \mathscr{E}(H) \rightarrow \mathscr{E}(H)$ a bijective map. If

$$
\Phi(\sqrt{A} B \sqrt{A})=\sqrt{\Phi(A)} \Phi(B) \sqrt{\Phi(A)}
$$

for all $A, B \in \mathscr{E}(H)$, then there exists an unitary or an antiunitary operator $U$ on $H$ such that $\Phi(A)=U A U^{*}$ for every $A \in \mathscr{E}(H)$.

Proof of Theorem 3. By Theorem 2 we know that $\Phi$ preserves projections in both directions, $\Phi(I)=I, \Phi(0)=0$, and $\Phi$ is orthoadditive on $\mathscr{P}(H)$.

Furthermore, $\Phi$ preserves the order of projections in both directions. To see this, let $P, Q \in \mathscr{P}(H)$. It is clear that

$$
\begin{gathered}
P \leq Q \Longleftrightarrow P Q=Q P=P \Longleftrightarrow Q P Q=P, \quad P Q P=P \\
\Longleftrightarrow Q^{\alpha} P^{\beta} Q^{\alpha}=P^{\beta}=P, \quad P^{\alpha} Q^{\beta} P^{\alpha}=P^{\alpha}=P .
\end{gathered}
$$

So, $P \leq Q$ implies that

$$
\begin{aligned}
& \Phi(P)=\Phi\left(Q^{\alpha} P^{\beta} Q^{\alpha}\right)=\Phi(Q)^{\alpha} \Phi(P)^{\beta} \Phi(Q)^{\alpha}, \\
& \Phi(P)=\Phi\left(P^{\alpha} Q^{\beta} P^{\alpha}\right)=\Phi(P)^{\alpha} \Phi(Q)^{\beta} \Phi(P)^{\alpha} .
\end{aligned}
$$

Consequently, $\Phi(P) \leq \Phi(Q)$. Similarly, using $\Phi^{-1}$, one can check that $\Phi(P) \leq \Phi(Q)$ implies that $P \leq Q$.

So, by Theorem 2 and [9], the restriction of $\Phi$ on $\mathscr{P}(H)$ can be extended to a bounded linear map $\Psi: \mathscr{B}(H) \rightarrow$ $\mathscr{B}(H)$. Note that $\Psi$ preserves projections in both directions; it must be a Jordan star-isomorphism. Since every Jordan star-automorphism of $\mathscr{B}(H)$ is either a star-automorphism or star-antiautomorphism, there exists a unitary operator $U$ or an antiunitary operator $V$ such that

$$
\Psi(A)=U A U^{*} \quad \forall A \in \mathscr{B}(H)
$$

or

$$
\Psi(A)=V A^{*} V^{*} \quad \forall A \in \mathscr{B}(H) .
$$

It follows that there is a unitary or an antiunitary operator $U$ such that $\Phi(P)=U P U^{*}$ for all $P \in \mathscr{P}(H)$.

Without loss of generality, we can assume that $\Phi(P)=P$ holds for every projection $P$.

Next we show that $\Phi(\lambda P)=\lambda P$ holds for every $\lambda \in[0,1]$ and $P \in \mathscr{P}(H)$. 
By the definition of $\Phi$ that we have for any $\lambda \in[0,1]$ and any rank one projection $P$, we have

$$
\Phi(\lambda P)=\Phi\left(P^{\alpha}\left(\lambda^{1 / \beta} P\right)^{\beta} P^{\alpha}\right)=P \Phi\left(\lambda^{1 / \beta} P\right)^{\beta} P .
$$

So there is a $f_{P}(\lambda) \in[0,1]$ such that $\Phi(\lambda P)=f_{P}(\lambda) P$. Denote $f_{P}$ by $f$; that is, $f(\lambda)=f_{P}(\lambda) . f:[0,1] \rightarrow[0,1]$ is a function such that $\Phi(\lambda P)=f(\lambda) P$. It follows that for every $\mu \in[0,1]$

$$
\begin{aligned}
\Phi(\lambda \mu P) & =f(\lambda \mu) P, \\
\Phi(\lambda \mu P) & =\Phi\left(\lambda^{(1 / 2 \alpha) \alpha} P \mu^{(1 / \beta) \beta} P \lambda^{(1 / 2 \alpha) \alpha} P\right) \\
& =\Phi\left(\lambda^{1 / 2 \alpha} P\right)^{\alpha} \Phi\left(\mu^{1 / \beta} P\right)^{\beta} \Phi\left(\lambda^{1 / 2 \alpha} P\right)^{\alpha} \\
& =f\left(\lambda^{1 / 2 \alpha}\right)^{2 \alpha} f\left(\mu^{1 / \beta}\right)^{\beta} P .
\end{aligned}
$$

On the other hand,

$$
\begin{gathered}
f(\lambda) P=\Phi(\lambda P)=\Phi\left(\lambda^{(1 / 2 \alpha) \alpha} P P P \lambda^{(1 / 2 \alpha) \alpha}\right)=f\left(\lambda^{1 / 2 \alpha}\right)^{2 \alpha} P, \\
f(\mu) P=\Phi(\mu P)=\Phi\left(I \mu^{(1 / \beta) \beta} P I\right)=f\left(\mu^{1 / \beta}\right)^{\beta} P .
\end{gathered}
$$

Thus $f\left(\lambda^{1 / 2 \alpha}\right)^{2 \alpha}=f(\lambda)$ and $f\left(\mu^{1 / \beta}\right)^{\beta}=f(\mu)$. Hence, we have $f(\lambda \mu)=f(\lambda) f(\mu)$ holds for all $\lambda, \mu \in[0,1]$; that is, $f$ is multiplicative.

Obviously, by the surjectivity of $\Phi, f([0,1])=[0,1]$. So $f$ is continuous on $[0,1]$. It is well known that every multiplicative continuous bijection $g:[0,1] \rightarrow[0,1]$ is of the form $g(\lambda)=\lambda^{\rho}(\rho>0)$. Hence there is a $\rho_{P}>0$ such that $f(\lambda)=\lambda^{\rho_{P}}$ for every $\lambda \in[0,1]$. It follows that

$$
\Phi(\lambda P)=\lambda^{\rho_{P}} P
$$

for every rank one projection $P$.

We will show that $\rho_{P}$ is independent of $P$. For any rank one projections $P, Q$ and $\lambda, \mu \in[0,1]$, we have

$$
\begin{aligned}
\Phi\left(\lambda P \mu^{2} Q \lambda P\right) & =\Phi\left(\lambda^{(1 / \alpha) \alpha} P \mu^{(2 / \beta) \beta} Q \lambda^{(1 / \alpha) \alpha} P\right) \\
& =f\left(\lambda^{1 / \alpha}\right)^{2 \alpha} f\left(\mu^{2 / \beta}\right)^{\beta} P Q P=\lambda^{2 \rho_{P}} \mu^{2 \rho_{Q}} P Q P \\
\Phi\left(\mu P \lambda^{2} Q \mu P\right) & =\Phi\left(\mu^{(1 / \alpha) \alpha} P \lambda^{(2 / \beta) \beta} Q \mu^{(1 / \alpha) \alpha} P\right) \\
& =\mu^{2 \rho_{P}} \lambda^{2 \rho_{Q}} P Q P .
\end{aligned}
$$

If $P Q \neq 0$, we get

$$
\lambda^{2 \rho_{P}} \mu^{2 \rho_{\mathrm{Q}}}=\mu^{2 \rho_{P}} \lambda^{2 \rho_{\mathrm{Q}}} .
$$

Letting $\mu=1$ and comparing two sides of the above equation yield $\lambda^{\rho_{P}}=\lambda^{\rho_{Q}}$. So $\rho_{P}=\rho_{Q}$. If $P Q=0$, pick a rank one projection $R$ so that $P R P \neq 0$ and $Q R Q \neq 0$. Then $\Phi(\lambda R)=$ $\lambda^{\rho_{R}} R$. By the fact we just checked, we get $\rho_{P}=\rho_{R}=\rho_{Q}$. It follows that there is a common $\rho \geq 0$ such that

$$
\Phi(\lambda P)=\lambda^{\rho} P
$$

holds for all rank one projection $P$ and $\lambda \in[0,1]$.
We claim that $\rho=1$. To see this, it is enough to check that $f(\lambda+\mu)=f(\lambda)+f(\mu)$ whenever $\lambda+\mu \leq 1$, where $f(\lambda)=\lambda^{\rho}$. Pick unit vectors $x, y$ with $\langle x, y\rangle=0$ and let $P=x \otimes x$, $Q=y \otimes y$, and $A=\left(\lambda^{1 / 2} x+\mu^{1 / 2} y\right) \otimes\left(\lambda^{1 / 2} x+\mu^{1 / 2} y\right)$. Then

$$
\begin{aligned}
A P A= & \left(\lambda^{1 / 2} x+\mu^{1 / 2} y\right) \otimes\left(\lambda^{1 / 2} x+\mu^{1 / 2} y\right) x \\
& \otimes x\left(\lambda^{1 / 2} x+\mu^{1 / 2} y\right) \otimes\left(\lambda^{1 / 2} x+\mu^{1 / 2} y\right) \\
= & \lambda^{1 / 2}\left(\lambda^{1 / 2} x+\mu^{1 / 2} y\right) \otimes x\left(\lambda^{1 / 2} x+\mu^{1 / 2} y\right) \\
& \otimes\left(\lambda^{1 / 2} x+\mu^{1 / 2} y\right) \\
= & \lambda\left(\lambda^{1 / 2} x+\mu^{1 / 2} y\right) \otimes\left(\lambda^{1 / 2} x+\mu^{1 / 2} y\right)=\lambda A .
\end{aligned}
$$

Similarly, we have

$$
\begin{gathered}
A Q A=\mu A \\
A(P+Q) A=A^{2}=(\lambda+\mu) A .
\end{gathered}
$$

Since $\Phi$ is orthoadditive on $\mathscr{P}(H)$, we have

$$
\begin{aligned}
\Phi(A(P+Q) A)= & \Phi\left(A^{(1 / \alpha) \alpha}(P+Q)^{(1 / \beta) \beta} A^{(1 / \alpha) \alpha}\right) \\
= & \Phi\left(A^{1 / \alpha}\right)^{\alpha} \Phi\left((P+Q)^{1 / \beta}\right)^{\beta} \Phi\left(A^{1 / \alpha}\right)^{\alpha} \\
= & \Phi\left(A^{1 / \alpha}\right)^{\alpha} \Phi(P+Q) \Phi\left(A^{1 / \alpha}\right)^{\alpha} \\
= & \Phi\left(A^{1 / \alpha}\right)^{\alpha}(\Phi(P)+\Phi(Q)) \Phi\left(A^{1 / \alpha}\right)^{\alpha} \\
= & \Phi\left(A^{1 / \alpha}\right)^{\alpha} \Phi\left(P^{1 / \beta}\right)^{\beta} \Phi\left(A^{1 / \alpha}\right)^{\alpha} \\
& +\Phi\left(A^{1 / \alpha}\right)^{\alpha} \Phi\left(Q^{1 / \beta}\right)^{\beta} \Phi\left(A^{1 / \alpha}\right)^{\alpha} \\
= & \Phi(A P A)+\Phi(A Q A) .
\end{aligned}
$$

Note that $A_{0}=A /\|A\|$ is a rank one projection, and for any $\delta \in[0,1]$, we have

$$
\begin{aligned}
\Phi(\delta A) & =\Phi\left(\delta\|A\| A_{0}\right)=f(\delta\|A\|) A_{0} \\
& =f(\delta) f(\|A\|) \Phi\left(A_{0}\right)=f(\delta) \Phi(A)
\end{aligned}
$$

Thus $\Phi(\delta A)=f(\delta) \Phi(A)$ and

$$
\begin{aligned}
f(\lambda+\mu) \Phi(A) & =\Phi((\lambda+\mu) A)=\Phi(A(P+Q) A) \\
& =\Phi(A P A)+\Phi(A Q A) \\
& =\Phi(\lambda A)+\Phi(\mu A) \\
& =f(\lambda) \Phi(A)+f(\mu) \Phi(A) .
\end{aligned}
$$

This forces that $f(\lambda+\mu)=f(\lambda)+f(\mu)$ whenever $\lambda+\mu \leq 1$, and hence, $\rho=1$.

Now, let us show that $\Phi(\lambda P)=\lambda P$ holds for all $\lambda \in[0,1]$ and all $P \in \mathscr{P}(H)$. By the orthoadditivity of $\Phi, \Phi(\lambda P)=\lambda P$ holds for every $\lambda \in[0,1]$ and every finite rank projection 
$P \in \mathscr{P}(H)$. Assume $P$ have infinite rank. For any $x \in \operatorname{ran}(P)$ with $\|x\|=1$, we have

$$
\begin{gathered}
\Phi\left(\lambda^{\alpha} P x \otimes x \lambda^{\alpha} P\right)=\Phi(\lambda P)^{\alpha} x \otimes \Phi(\lambda P)^{\alpha} x \\
\Phi\left(\lambda^{\alpha} P x \otimes x \lambda^{\alpha} P\right)=\Phi\left(\lambda^{2 \alpha} x \otimes x\right)=\lambda^{2} x \otimes x .
\end{gathered}
$$

It follows that $\Phi(\lambda P)^{\alpha} x$ and $x$ are linearly dependent; that is, there is a scalar $f_{x}(\lambda)$, dependent on $x$ such that $\Phi(\lambda P)^{\alpha} x=$ $f_{x}(\lambda) x$ with $f_{x}(0)=0$ and $f_{x}(1)=1$. Similarly, for every $y \in \operatorname{ran}(I-P)$, we have $\Phi(\lambda P)^{\alpha} y=0$. These entail that there exists a function $g_{P}(x, \lambda)$ having values in $[0,1]$ and satisfying $\Phi(\lambda P) x=f_{x}(\lambda)^{1 / \alpha} x=g_{P}(x, \lambda) P x$ for all $x \in \operatorname{ran} P$ and all $x \in(\operatorname{ran} P)^{\perp}$. Hence, $g_{P}(x, 0)=0$ and $g_{P}(x, 1)=1$. As before, for a fixed $x \in \operatorname{ran} P$, we have that $g_{P}(x, \lambda)=\lambda^{\delta}$ for some positive $\delta$. Next we claim that $g(x, \lambda)=\lambda$. Taking the rank one projection $Q=x \otimes x$ for $x \in \operatorname{ran} P$, so $Q \leq P$, we have

$$
\lambda^{\delta} Q=\Phi(Q(\lambda P) Q)=\Phi\left(\lambda^{1 / 2} Q P\left(\lambda^{1 / 2} Q\right)\right)=\lambda Q
$$

which entails that $\lambda^{\delta}=\lambda$. In sum, now we have that $\Phi(\lambda P) x=$ 0 for $x \in(\operatorname{ran} P)^{\perp}$ and $\Phi(\lambda P) x=\lambda P x=\lambda x$ for $x \in \operatorname{ran} P$. Let $H=\operatorname{ran} P \oplus(\operatorname{ran} P)^{\perp}$, since $\Phi(\lambda P) \geq 0$,

$$
\Phi(\lambda P)=\left(\begin{array}{ll}
\lambda & 0 \\
0 & 0
\end{array}\right)=\lambda P
$$

Finally, we show that $\Phi(A)=A$ for every $A \in \mathscr{E}(H)$. In fact, letting $A \in \mathscr{E}(H)$, for every unit vector $x \in H$, we have

$$
\begin{aligned}
\Phi(A)^{\alpha} x \otimes \Phi(A)^{\alpha} x & =\Phi\left(A^{\alpha}\right) x \otimes x \Phi\left(A^{\alpha}\right) \\
& =\Phi\left(A^{\alpha}\right)(x \otimes x)^{\beta} \Phi\left(A^{\alpha}\right) \\
& =\Phi\left(A^{\alpha}(x \otimes x)^{\beta} A^{\alpha}\right) \\
& =A^{\alpha} x \otimes A^{\alpha} x .
\end{aligned}
$$

Thus there exists a scalar $\lambda_{x}$ with $\left|\lambda_{x}\right|=1$ such that $\Phi(A)^{\alpha} x=$ $\lambda_{x} A x$. This entails that $\Phi(A)^{\alpha}=c A$ for some scalar $c$ with $|c|=1$. Since both $A$ and $\Phi(A)$ are positive, we see that $c=1$. Hence $\Phi(A)^{\alpha}=A^{\alpha}$ and the uniqueness of the positive root of a positive operator implies that $\Phi(A)=A$, as desired.

\section{Conflict of Interests}

The authors declare that there is no conflict of interests regarding the publication of this paper.

\section{Acknowledgments}

This work was supported partially by National Science Foundation of China (11201329, 11171249, and 11271217).

\section{References}

[1] G. Ludwig, Foundations of Quantum Mechanics, vol. 1, Springer, Berlin, Germany, 1983.
[2] J. Hou, K. He, and X. Qi, "Characterizing sequential isomorphisms on Hilbert space effect algebras," Journal of Physics A: Mathematical and Theoretical, vol. 43, no. 31, Article ID 315206, 2010.

[3] K. He, J.-C. Hou, and C.-K. Li, "A geometric characterization of invertible quantum measurement maps," Journal of Functional Analysis, vol. 264, no. 2, pp. 464-478, 2013.

[4] S. O. Kim, "Automorphisms of Hilbert space effect algebras," Linear Algebra and Its Applications, vol. 402, pp. 193-198, 2005.

[5] L. Molnár, "On some automorphisms of the set of effects on Hilbert space," Letters in Mathematical Physics, vol. 51, no. 1, pp. $37-45,2000$.

[6] L. Molnár, "Preservers on Hilbert space effects," Linear Algebra and Its Applications, vol. 370, pp. 287-300, 2003.

[7] L. Molnár and P. Šemrl, "Conditional affine and condional sequential automorphisms of the set of Hilbert space effect algebras," In press.

[8] L. Molnár, Selected Preserver Problems on Algebraic Structures of Linear Operators and on Function Spaces, vol. 1895 of Lecture Notes in Mathematics, Springer, 2007.

[9] L. J. Bunce and J. D. M. Wright, “The Mackey-Gleason problem," Bulletin of the American Mathematical Society, vol. 26, no. 2, pp. 288-293, 1992. 


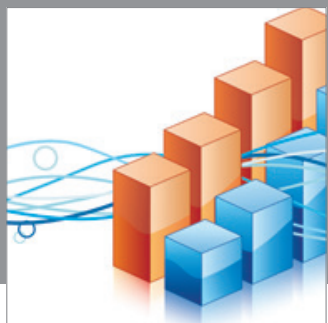

Advances in

Operations Research

mansans

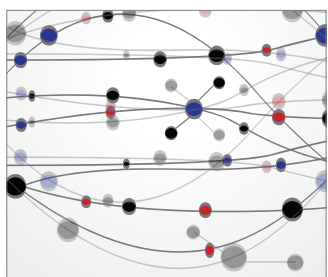

The Scientific World Journal
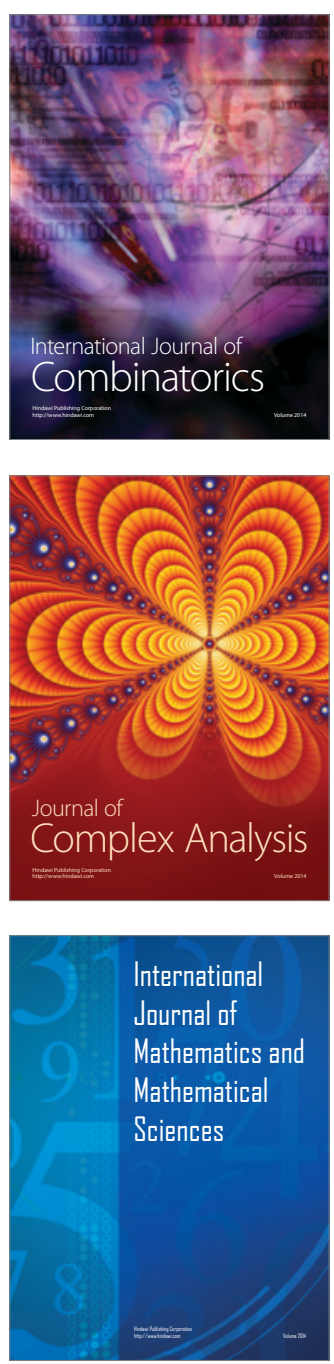
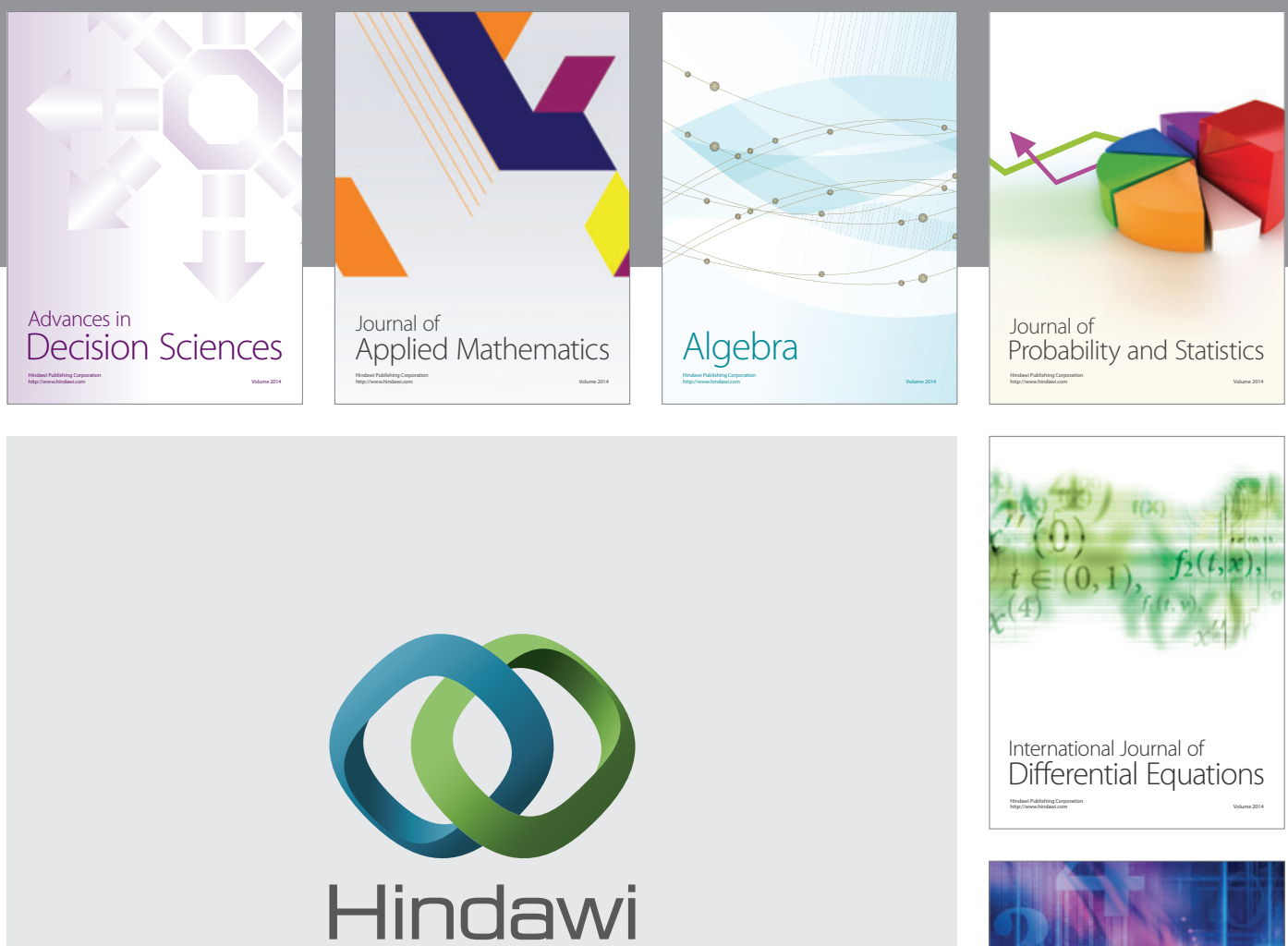

Submit your manuscripts at http://www.hindawi.com
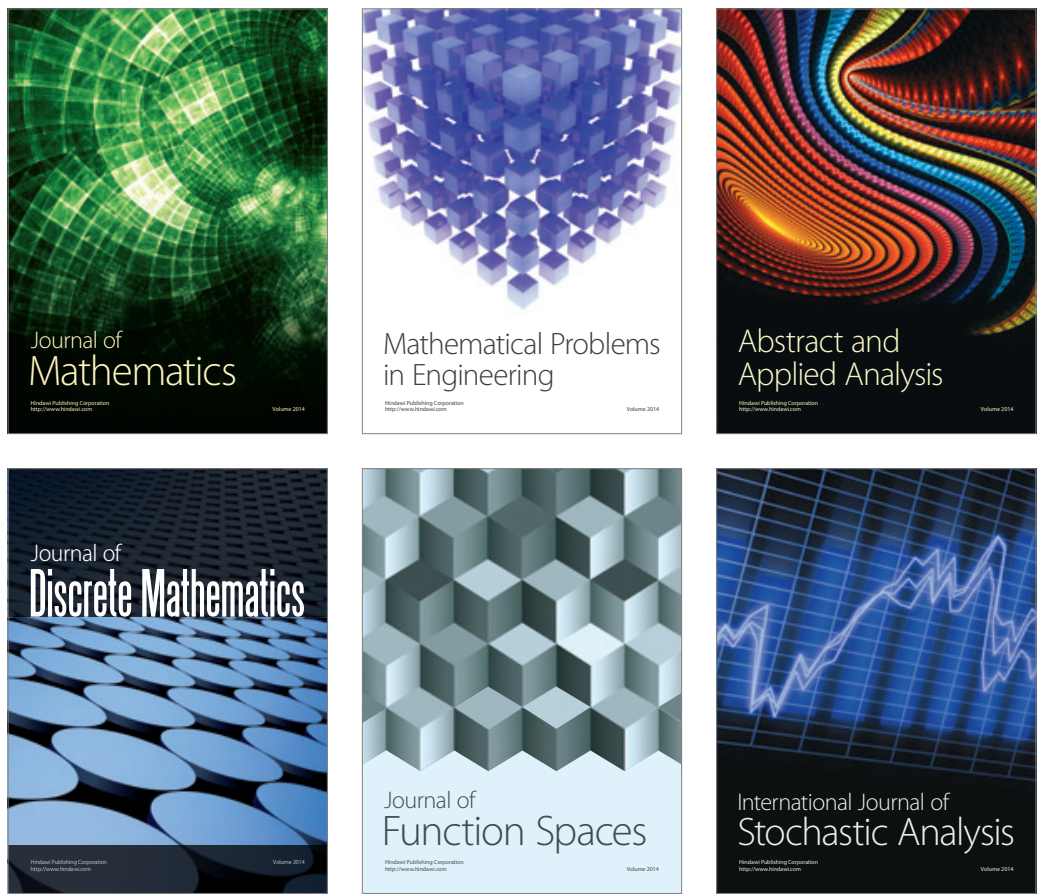

Journal of

Function Spaces

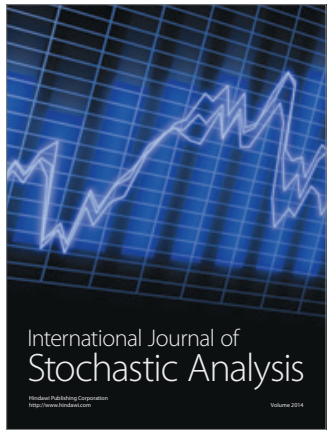

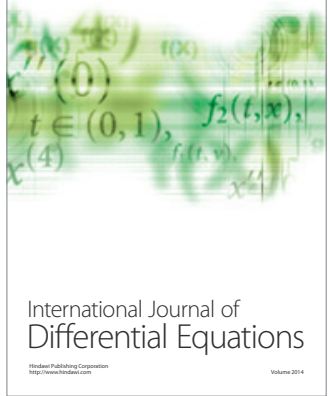
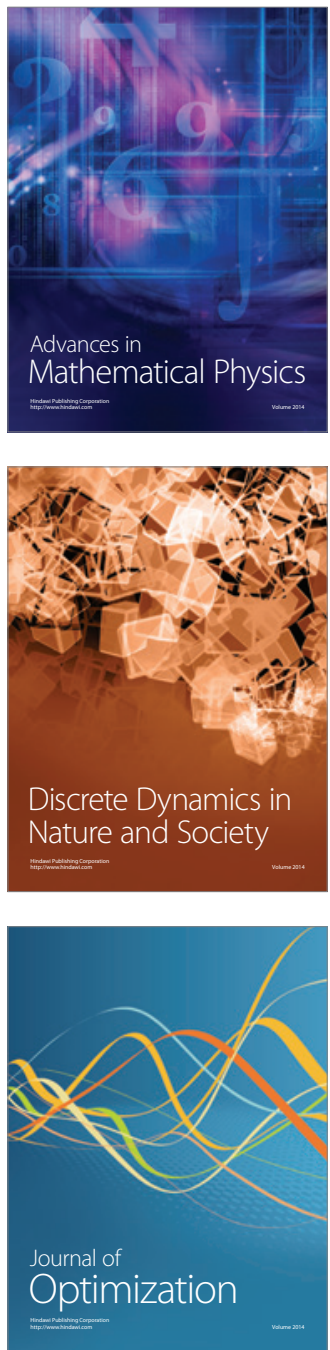